\title{
Mucoadhesive Paclitaxel Formulation
}

National Cancer Institute

\section{Source}

National Cancer Institute. Mucoadhesive Paclitaxel Formulation. NCI Thesaurus. Code C107188.

An orally available, mucoadhesive lipid preparation consisting of paclitaxel, a compound extracted from the Pacific yew tree Taxus brevifolia, in a formulation that is comprised of a mixture of monoolein, tricarprylin, and Tween 80 , with potential antineoplastic activity. Upon oral administration, DHP107 forms droplets and micelles in the intestine; these adhere to mucoepithelial cells in the gastrointestinal tract and are absorbed through lipidbased uptake mechanisms. Upon absorption, paclitaxel binds to and stabilizes tubulin molecules, which results in the inhibition of both microtubule depolymerization and cell division. This agent also induces apoptosis by both binding to and blocking the function of the apoptosis inhibitor protein B-cell Leukemia 2 (Bcl-2).The mucoadhesive paclitaxel formulation does not contain P-glycoprotein inhibitors, the solvent cremophor or any other toxic solvent. 